\title{
On the Problem of Detection and Discrimination of Double Talk and Change in the Echo Path
}

\author{
C.Carlemalm, F.Gustafsson ${ }^{\dagger}$ B.Wahlberg ${ }^{\ddagger}$
}

\section{Abstract}

The problem of detection and discrimination of double talk and change in the echo path in a telephone channel is considered. A change in echo path requires fast adaptation of the channel model to be able to equalize the echo dynamics. On the other hand, the adaption rate should be reduced when double talk occurs. Thus, it is critical to quickly detect a change in echo path while not confusing it with double talk, which gives a similar effect. The proposed likelihood based approach compares a global channel model with a local one over a sliding window, both estimated with the Recursive Least Squares algorithm.

\section{Introduction}

The purpose of this paper is to study the problem of detection and discrimination of double talk and change in the echo path of a signal. With use of statistical estimation and detection algorithms suggested in [1], the posed problem is analyzed.

The telephone network of today generates undesired echoes within and near the end of telephone connections. The echo is of course annoying and makes the conversation difficult. To control and reduce the audible echoes is therefore an important task.

Any wire which suffers from a significant transmission delay is potentially liable to cause audible echoes. An international telephone conversation will due to the large distance cause a delay of at least $500 \mathrm{~ms}$ [2]. The two parts of the telephone system are connected via an exchange (a Group Switching Center, GSC, in U.K.) [3]. This exchange includes a 2-wire-to-4-wire hybrid which is based on the principle of the Wheatstone bridge. Unfortunately it is not economically possible to keep the impedances in this component balanced. This results in a leakage of signal power which might be heard as an annoying echo when there is a large transmission delay between the two participants in the telephone conversation.

Echo control is also a prominent problem in the new digital mobile telephone systems since the combination of speech coding, channel coding and signal processing involves considerable delays comparable with those of long-distance international calls. When a mobile telephone is connected to an ordinary telephone, audible echoes are due to the involved 4-to-2-wire hybrid very plausible to occur. Echo control is today required by a CCITT recommendation.

The echo cancelers that are used today consist of an adaptive FIR-filter with typically 512 filter coefficients [4].

The schematic of a typical echo canceler is depicted in figure 1 . The undesired echo is denoted $y$. The echo canceler generates the echo replica, $\hat{y}$, by applying the known reference signal, $\varphi$, to the FIR-filter. The remaining echo energy is used to improve the replica.

Thus, the idea of the echo canceler is to use a FIR-filter to approximate the transfer function of the echo and let the filter coefficients be adjusted based on the calculated prediction

* S3-Automatic Control, Royal Institute of Technology, S-100 44 Stockholm, Sweden, email: cath@elixir.e.kth.se

$\dagger^{\dagger}$ Automatic Control, Linköping University, S-581 83 Linköping, Sweden, email: fredrik@isy.liu.se

${ }^{\ddagger}$ S3-Automatic Control, Royal Institute of Technology, S-100 44 Stockholm, Sweden, email: bo@elixir.e.kth.se

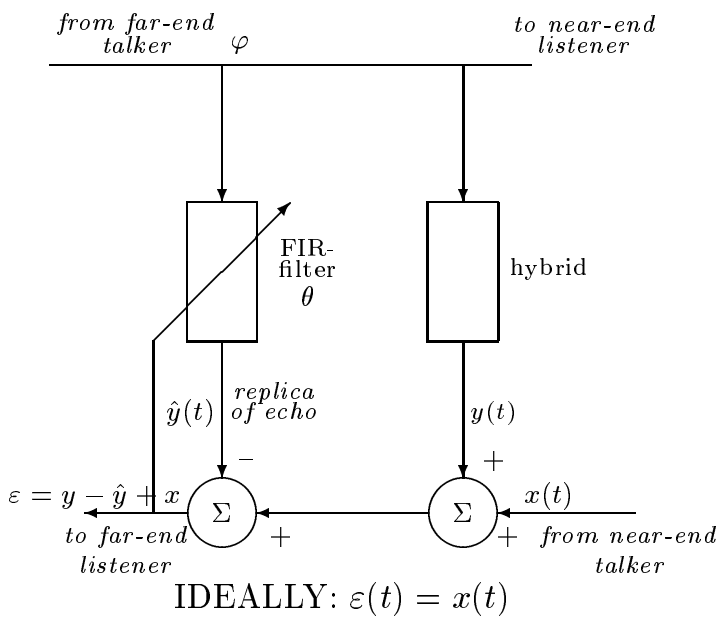

Figure 1: Schematic of an echo canceler.

error.

This simple solution to the problem of echo control is unfortunately not applicable due to the existence of so-called double talk, which also manifests itself in an abrupt increase of the prediction error.

Double talk is a phenomenon that occurs when both participants in the telephone conversation talk simultaneously. According to [5], double talk happens very frequently; the probability for double talk is about 20\%. Due to the increased distortion of the output signal, the coefficients of the echo canceler start varying and will diverge if they are not frozen, i.e. adaptation is stopped.

The conclusions to be made are that merely measuring the prediction error will not discriminate between double talk and echo and that the echo canceller must react differently whether double talk or echo path change has occured. Further, the detection and discimination algorithm must be fast in order to prevent the FIR-filter in the echo canceler from being misadjusted.

In this paper new and more advanced statistical methods will be applied on the posed problem.

This paper is organized as follows. In section 2 the model and the notation used in the following sections are introduced and the assumptions are made. Section 3 discusses the solution algorithm. Next, in section 4 some experimental results are presented. Finally, section 5 gives the conclusions and some remarks.

\section{Preliminaries}

In this section the notation and the model used in the following sections are introduced.

The model used to describe the dynamics of the output signal is given below. It is also shown in figure 2 .

Model:

$$
y(t)= \begin{cases}\varphi^{\prime}(t) \theta_{1}(t)+w_{1}(t), & \text { if } t \leq t_{0} \\ \varphi^{\prime}(t) \theta_{2}(t)+w_{2}(t), & \text { if } t>t_{0}\end{cases}
$$


known input signal at time $t, \varphi(t)=[-y(t-1),-y(t-$ $\left.2), \ldots,-y\left(t-n_{a}\right), u(t), u(t-1), \ldots, u\left(t-n_{b}\right)\right]^{\prime}, \theta_{j}(t)=$ $\left[\theta_{j}^{0}(t), \theta_{j}^{1}(t), \ldots, \theta_{j}^{n_{a}+n_{b}}(t)\right]^{\prime}, \hat{\theta}_{j}$ denotes the estimate of the parameter vector $\theta_{j}$ and $w_{j}(t)$ is zero mean white Gaussian noise.

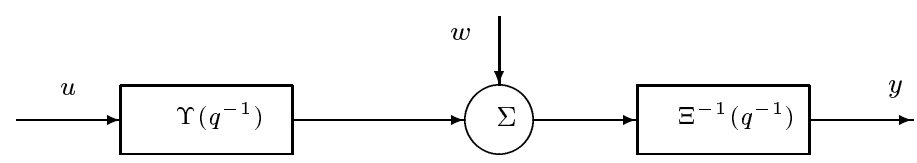

Figure 2: The model used to describe the signal. The output of the channel is given by the ARX-model $y(t)=-\theta_{j}^{0}(t) y(t-$ 1) $-\theta_{j}^{1}(t) y(t-2)-\ldots-\theta_{j}^{n_{a}-1}(t) y\left(t-n_{a}\right)+\theta_{j}^{n_{a+1}}(t) u(t)+$ $\theta_{j}^{n_{a}+2}(t) u(t-1)+\ldots+\theta_{j}^{n_{a}+n_{b}}(t) u\left(t-n_{b}\right)+w_{j}(t)$.

Consider the model (1) at time $t$ under the following different hypotheses about the parameter and noise variance before and after the possible abrupt change at time $t_{0}$ :

$$
\begin{array}{lll}
H_{0}: & \theta_{1}=\theta_{2} & \text { and } \operatorname{Var}\left(w_{1}\right)=\operatorname{Var}\left(w_{2}\right) \\
H_{1}: & \theta_{1} \neq \theta_{2} & \text { and } \operatorname{Var}\left(w_{1}\right)=\operatorname{Var}\left(w_{2}\right) \\
H_{2}: & \theta_{1}=\theta_{2} & \text { and } \operatorname{Var}\left(w_{1}\right) \neq \operatorname{Var}\left(w_{2}\right)
\end{array}
$$

The situation $H_{1}$ occurs when an echo path change happens at time $t_{0}$, the situation $H_{2}$ when double talk appears at time $t_{0}$ and finally the null hypothesis when neither echo path change nor double talk has occured at time $t_{0}$. We can assign a prior probability of each event as $q_{1}=P\left(H_{1}\right)$, $q_{2}=P\left(H_{2}\right)$ and $q_{0}=P\left(H_{0}\right)=1-q_{1}-q_{2}$. We assume that $P\left(H_{1} \cap H_{2}\right)=0$

The model (1) should be plausible under the assumption that the background noise is not too small.

\section{Analysis}

In this section the statistical estimation and detection algorithms that in this paper are used to solve the problem of detection and discrimination of double talk and change in the echo path are described.

\subsection{Estimation of the Involved Parameters}

The objective of this subsection is to estimate the parameter vector $\theta$ which was introduced in the previous section.

The choice of estimation algorithm is of course crucial since the detection method will rely on the estimates and the estimation algorithm affects the computational complexity.

The algorithm to estimate the parameter vector $\theta$ is in this paper chosen to be the Recursive Least Square algorithm (RLS) proposed in [6]. Its computational complexity is of the order $\mathrm{O}\left(n^{2}\right)$. There exists a modification of this algorithm, the so called fast RLS, which has a reduced complexity [7].

Algorithm 1 Consider the linear regression $y_{t}=\varphi_{t}^{\prime} \theta+w_{t}$, where $y_{t}$ and the regression vector $\varphi_{t}$ are known at time $t$, $\theta$ is the unknown parameter vector which is to be estimated and $w_{t}$ is white Gaussian noise with covariance matrix $\Lambda_{t}$.

Assume that the RLS estimate at time $t-1$ is $\theta_{t-1}$ with covariance matrix $P_{t-1}$. Then a new measurement gives the update formulas:

$$
\theta_{t}=\theta_{t-1}+P_{t-1} \varphi_{t}\left[\varphi_{t}^{\prime} P_{t-1} \varphi_{t}+\Lambda_{t}\right]^{-1}\left(y_{t}-\varphi_{t}^{\prime} \theta_{t-1}\right)
$$

The a posteriori distribution of $\theta$ is $N\left(\theta_{t}, P_{t}\right)$.

The RLS algorithm minimizes the loss function $V_{t}(\theta)=$ $\sum_{k=1}^{t}\left(y_{k}-\varphi_{k}^{\prime} \theta\right)^{\prime} \Lambda_{k}^{-1}\left(y_{k}-\varphi_{k}^{\prime} \theta\right)$ with respect to $\theta$.

\subsection{Algorithm for Detection and Discrimination}

In this section the likelihood functions corresponding to the different hypotheses, that were stated in section 2 , are determined. We propose a sequential detection approach using a sliding window. The involved quantities are defined below:

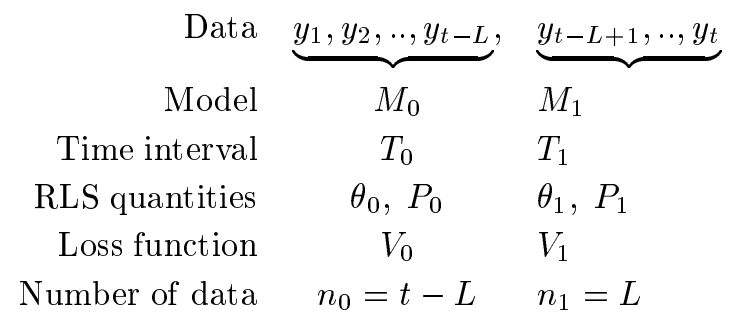

The loss functions are defined by

$$
V_{j}(\theta)=\sum_{k \in T_{j}}\left(y_{k}-\varphi_{k}^{\prime} \theta\right)^{\prime} \Lambda_{k}^{-1}\left(y_{k}-\varphi_{k}^{\prime} \theta\right)
$$

The algorithm is described below.

\section{Algorithm 2 .}

0 . Choose the sliding window length $L$ and the model structure, that is, the elements of $\varphi(t)$.

1. Estimate recursively $\theta_{0}$ and its covariance matrix $P_{0}$ based on $\left\{y_{t}: t \in T_{0}\right\}$, where $T_{0}=\{1,2, \ldots, t-L\}$.

2. Estimate recursively $\theta_{1}$ and its covariance matrix $P_{1}$ based on $\left\{y_{t}: t \in T_{1}\right\}$, where $T_{1}=\left\{y_{t-L+1}, y_{t-L+2}, \ldots, y_{t}\right\}$.

3. Compute the likelihood function $l_{i}$, to be defined below, corresponding to hypothesis $i, i=0,1,2$.

4. Compare the likelihood functions and use a stopping rule, discussed below, to decide whether $H_{1}$ or $H_{2}$ has occured at time instant $t-L$.

5. If the null hypothesis is rejected, restart the algorithm by letting $t=0$.

6. Let $t=t+1$ and iterate the steps 1-6.

The maximum likelihood approach is a standard technique often used in statistical detection theory. We will consider the, in this problem, slightly more general maximum a posteriori approach, where the prior probabilities $q_{i}$ for each hypothesis can be incorporated. Using results in [1], the exact a posteriori probabilities

$$
l_{i}=-2 \log p\left(H_{i} \mid y_{1}, y_{2}, \ldots, y_{t}\right),
$$

are accurately approximated for large $t$ and $L, t \gg L$ by:

$$
\begin{aligned}
l_{0}= & \left(n_{0}+n_{1}-2\right) \log \frac{V_{0}\left(\theta_{0}\right)+V_{1}\left(\theta_{0}\right)}{n_{0}+n_{1}-4} \\
& -\log \operatorname{det}\left(P_{0}^{-1}+P_{1}^{-1}\right)+2 \log \left(q_{0}\right) \\
l_{1}= & \left(n_{0}+n_{1}-2\right) \log \frac{V_{0}\left(\theta_{0}\right)+V_{1}\left(\theta_{1}\right)}{n_{0}+n_{1}-4} \\
& -\log \operatorname{det} P_{0}-\log \operatorname{det} P_{1}+2 \log \left(q_{1}\right) \\
l_{2}= & \left(n_{0}-2\right) \log \frac{V_{0}\left(\theta_{0}\right)}{n_{0}-4}+\left(n_{1}-2\right) \log \frac{V_{1}\left(\theta_{0}\right)}{n_{1}-4} \\
& -2 \log \operatorname{det} P_{0}+2 \log q_{2}
\end{aligned}
$$




$$
\theta_{t o t}=\left(P_{0}^{-1}+P_{1}^{-1}\right)^{-1}\left(P_{0}^{-1} \theta_{0}+P_{1}^{-1} \theta_{1}\right)
$$

is the parameter estimate corresponding to all available data. The negative log likelihood can be derived from the expressions above by letting the prior being non-informative, that is $q_{i}=1 / 3$.

\subsection{Stopping rule}

To conclude which hypothesis is valid at a specific time instant, the values of the likelihood functions are calculated. The hypothesis corresponding to the least value is assumed to hold.

In real-time applications, the alarm time is the crucial quantity. Of course, it should be as small as possible. The most natural stopping rule is to say that a change has occured as soon as $l_{1}$ or $l_{2}$ is smaller than $l_{0}$. However, in order to avoid false alarms, it might be advisable to accept a change hypothesis only if its likelihood has been the smallest one for a number of consequtive samples.

In methodology investigations it could be interesting to estimate the change time as accurately as possible. We advice to try to find the peak value of the likelihood as a function of time by requiring that $l_{1}-l_{0}$ (or $l_{2}-l_{0}$ ) has been decreasing for at least half a window length, $L$, and then starts to increase. This is the stopping rule examined in the next section.

\section{Experimental Results}

In this section the method which has been proposed in this paper for detection and discrimination of double talk (DT) and the echo path change (EPC) is evaluated by simulations using a real speech signal of length 6000 samples as input signal. The sampling frequency is $8000 \mathrm{~Hz}$.

First the input signal is filtered through two different impulse responses, which are measured impulse responses from real hybrids. The impulse responses used are shown in figure 3 .

To generate the different hypotheses, the signal is broken
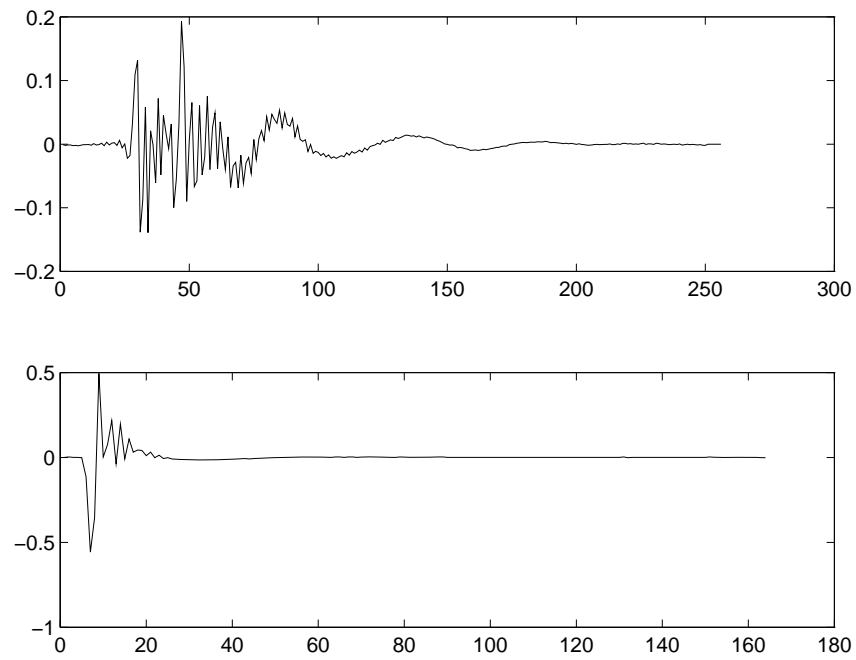

Figure 3: Impulse responses used in the simulations

up into segments. A change in the echo path is simulated by using different impulse responses in the different segments. By adding speech to the filtered input signal, double talk is simulated. The signal to noise ratio is approximately $15 \mathrm{~dB}$.

The results for a number of different cases are summarized below. In all cases, a change occurs at sample 1000, the window length is chosen to be 250 , a non-informative prior is

\begin{tabular}{|l|l|l|c|c|cc|}
\hline Hyp. & Model & No & Jump & Mean & \multicolumn{2}{|c|}{ Classification } \\
& jump & & jump & EPC & DT \\
\hline EPC & ARX(10) & $0 \%$ & $100 \%$ & 2408 & $100 \%$ & $0 \%$ \\
\hline DT & ARX(5) & $0 \%$ & $100 \%$ & 4291 & $0 \%$ & $100 \%$ \\
\hline EPC & ARX(5) & $0 \%$ & $100 \%$ & 2430 & $100 \%$ & $0 \%$ \\
\hline
\end{tabular}

By studying the examples above, it becomes clear that the detection and discrimination algorithm suggested in this paper is rather trustworthy.

The mean time delay for detection of an abrupt change is never more than 4000 samples. Thus, the delay is less than $0.5 \mathrm{~s}$, since the sampling frequency used is $8000 \mathrm{~Hz}$. When the window length is chosen to be 150, the probabilty for jump is reduced and the mean jump is increased approximately 100 samples.

\section{Conclusions}

The performance of the algorithm for detection and discrimination of double talk and change in the echo path suggested in this paper is experimentally verified in the previous section.

The selection of window length, $L$, is obviously crucial. The approximations of the a posteriori probabilities, $l_{i}$, in section 3 are based on the fact that $L$ is large.

The computational complexity of the proposed algorithm is quite high due to the used Recursive Least Squares algorithm which has a complexity of the order $\mathrm{O}\left(n^{2}\right)$. In fact, the total complexity of the suggested detection method is equivalent to the complexity of two parallel RLS filters. A different estimation algorithm could have been used but that would probably have affected the accuracy of the detection and discrimination algorithm.

\section{References}

[1] F. Gustafsson, Estimation of Discrete Parameters in Linear Systems, Ph.D. dissertation, Linköping University, Department of Electrical Engineering, Linköping, Sweden, 1992.

[2] K. Feher, Advanced Digital Communications. Englewood Cliffs, N.J.: Prentice Hall, 1987.

[3] R.H. Moffett, "Echo and Delay Problems in Some Digital Communication Systems", IEEE Communication Magazine, vol. 25, no.8, pp.41-47, 1987.

[4] N.A.M. Verhoeckx, H.C. van den Elzen, F.A.M. Snijders and P.J. van Gerwen, "Digital Echo Cancellation for Baseband Data Transmission", IEEE Transaction on Acoustics, Speech and Signal Processing, vol. ASSP-27, no.6, pp.768$781,1979$.

[5] M.M. Sondhi and D.A. Berkley, "Silence Echoes on the Telephone Network", Proc. of the IEEE, vol.68, no.8, pp.948963, 1980.

[6] L. Ljung and T. Söderström, Theory and Practice of Recursive Identification. MIT Press, Cambridge MA, 1983.

[7] C.W.K. Gritton, D.W. Lin, "Echo Cancellation Algorithms", IEEE ASSP Magazine, pp.30-38, 1984. 